\title{
BMJ Open Theory-driven group-based complex intervention to support self-management of osteoarthritis and low back pain in primary care physiotherapy: protocol for a cluster randomised controlled feasibility trial (SOLAS)
}

\author{
Deirdre A Hurley, ${ }^{1}$ Amanda M Hall, ${ }^{2}$ Laura Currie-Murphy, ${ }^{3}$ Tamar Pincus, ${ }^{4}$ \\ Steve Kamper, ${ }^{5}$ Chris Maher, ${ }^{5}$ Suzanne M McDonough, ${ }^{6}$ Chris Lonsdale, ${ }^{7}$ Nicola \\ E Walsh, ${ }^{8}$ Suzanne Guerin, ${ }^{9}$ Ricardo Segurado, ${ }^{10}$ James Matthews, ${ }^{1}$ SOLAS Trial \\ team
}

To cite: Hurley DA, Hall AM, Currie-Murphy L, et al. Theory-driven group-based complex intervention to support self-management of osteoarthritis and low back pain in primary care physiotherapy: protocol for a cluster randomised controlled feasibility trial (SOLAS). BMJ Open 2016;6:e010728. doi:10.1136/bmjopen-2015010728

- Prepublication history and additional material is available. To view please visit the journal (http://dx.doi.org/ 10.1136/bmjopen-2015010728).

Received 1 December 2015 Revised 7 December 2015 Accepted 8 December 2015

CrossMark

For numbered affiliations see end of article.

Correspondence to Dr Deirdre A Hurley; deirdre.hurleyosing@ucd.ie

\section{ABSTRACT}

Introduction: International clinical guidelines consistently endorse the promotion of selfmanagement (SM), including physical activity for patients with chronic low back pain (CLBP) and osteoarthritis $(O A)$. Patients frequently receive individual treatment and advice to self-manage from physiotherapists in primary care, but the successful implementation of a clinical and cost-effective group SM programme is a key priority for health service managers in Ireland to maximise long-term outcomes and efficient use of limited and costly resources.

Methods/analysis: This protocol describes an assessor-blinded cluster randomised controlled feasibility trial of a group-based education and exercise intervention underpinned by self-determination theory designed to support an increase in SM behaviour in patients with CLBP and OA in primary care physiotherapy. The primary care clinic will be the unit of randomisation (cluster), with each clinic randomised to 1 of 2 groups providing the Self-management of Osteoarthritis and Low back pain through Activity and Skills (SOLAS) intervention or usual individual physiotherapy. Patients are followed up at 6 weeks, 2 and 6 months. The primary outcomes are the (1) acceptability and demand of the intervention to patients and physiotherapists, (2) feasibility and optimal study design/procedures and sample size for a definitive trial. Secondary outcomes include exploratory analyses of: point estimates, $95 \% \mathrm{Cls}$, change scores and effect sizes in physical function, pain and disability outcomes; process of change in target SM behaviours and selected mediators; and the cost of the intervention to inform a definitive trial.

Ethics/dissemination: This feasibility trial protocol was approved by the UCD Human Research EthicsSciences Committee (LS-13-54 Currie-Hurley) and research access has been granted by the Health

\section{Strengths and limitations of this study}

- This protocol describes a cluster randomised controlled feasibility trial of a theory-driven group-based intervention for primary care patients with low back pain and osteoarthritis.

- This study will explore the acceptability and demand of the group-based intervention to patients and physiotherapists compared with usual individual physiotherapy.

- The exclusion of patients who are unwilling or unavailable to attend a 6 -week group programme will limit the study's generalisability.

Services Executive Primary Care Research Committee in January 2014. The study findings will be disseminated to the research, clinical and health service communities through publication in peerreviewed journals, presentation at national and international academic and clinical conferences. Trial registration number: ISRCTN 49875385; Pre-results.

\section{INTRODUCTION}

Chronic musculoskeletal pain conditions are the leading cause of disability globally, notably low back pain (LBP) ranked first, ${ }^{1}$ and hip and knee osteoarthritis (OA) ranked 11th in the Global Burden of Disease 2010 study (see online supplementary file 1 ). ${ }^{2}$ The global point prevalence of LBP was estimated at $9.4 \%$, with OA hip and knee combined at $3.8 \%^{3}$; Ireland has slightly higher rates of $12 \%$ and $4.2 \%$, respectively. ${ }^{4} 5$ In the over $50 \mathrm{~s}$, OA is the leading cause of disability 
worldwide, including Ireland (prevalence rate 12.9\%), ${ }^{6}$ and is predicted to increase due to rising ageing populations and obesity levels. ${ }^{7}$ Both conditions place substantial demand on health systems accounting for $10-18 \%$ of consultations in primary care, ${ }^{8}$ and between $5.4 \%$ and $12.6 \%$ of total health expenditure. ${ }^{9}$ In Ireland, $35.5 \%$ of primary care consulters experience chronic non-cancer pain at a cost of $€ 5.34$ billion per year. ${ }^{10}$ The disability associated with LBP and $\mathrm{OA}$ also places significant burden on families, carers, economies and society through absenteeism, presenteeism, work disability and the need for additional social supports. ${ }^{11} 12$

International clinical practice guidelines consistently endorse the promotion of self-management (SM) for people with $\mathrm{OA}^{13-15}$ and chronic $\mathrm{LBP}^{1617}$ as an integral component of care, with important elements being education about the individual's chronic condition, its consequences, and its management and the uptake of evidence-based SM behaviours by participants; including physical activity, specific exercise, and pharmacological and non-pharmacological approaches. ${ }^{15} 16$

While SM is advocated by policymakers for chronic health conditions, ${ }^{7}{ }^{18-20}$ health service commissioners require robust evidence of its clinical and costeffectiveness prior to widespread implementation. Recent reviews suggest only small or equivocal effects for SM compared with other approaches for these conditions, ${ }^{21}{ }^{22}$ while group-based SM programmes with healthcare professional input showed some beneficial effects, further evidence of their clinical and costeffectiveness is needed. ${ }^{23}$ Within Ireland's primary care health system, the majority of patients with OA and chronic low back pain (CLBP) referred for physiotherapy (PT) are treated individually ${ }^{2425}$ in a diverse range of health settings by staff with varying degrees of expertise. ${ }^{26}$ Our recent rapid review found equivocal effectiveness for individual and group-based SM programmes with limited cost-effectiveness studies and failed to identify a group-based intervention for both OA hip/knee and CLBP. ${ }^{27}$ These conditions often present concurrently in those aged over 45 years, ${ }^{28}{ }^{29}$ and could be treated together in order to maximise outcomes and minimise inefficiencies. ${ }^{30}$ The successful implementation of an evidence-based group SM programme for CLBP and OA is a key priority for Ireland's PT managers in current resource constrained times. ${ }^{19}$

The multifaceted nature of SM interventions with several interacting components that need to be tailored to the individual, and underpinned by a collaborative relationship between the patient and healthcare professional within the local health service constitutes a complex intervention, ${ }^{31}$ notwithstanding the additional challenges of consistent delivery in a group format, across a diverse range of health settings with varying resources and facilities. ${ }^{32} 33$ It has been argued that the policy for group interventions to encourage SM in primary care has raced ahead of the evidence and there are many unanswered questions for practitioners and policymakers aiming to establish client groups in terms of their feasibility, effectiveness and costs. ${ }^{32}{ }^{33}$ Therefore, following the Medical Research Council (MRC) framework, ${ }^{31}{ }^{34}$ we developed the Self-management of OA and LBP through Activity and Skills (SOLAS) intervention that was acceptable and met the needs of Ireland's primary care service stakeholders and the current evidence which will be reported in detail in a separate paper. Applying the MRC framework, it is necessary to establish the feasibility of delivering the SOLAS intervention to PTs and patients with OA and CLBP to determine 'can it work?', 'does it work?' and 'will it work?' 35 in primary care centres in Ireland compared with usual individual PT care, considered the most appropriate comparison. Hence, a cluster randomised controlled trial (RCT) design was chosen to avoid contamination of the control group. ${ }^{36}$ This feasibility trial will also determine the most efficient and effective design and sample size for a main effectiveness trial and provide an estimate of the cost of delivering the intervention compared with usual PT.

Consistent with the recommendations of trial reporting guidelines, ${ }^{37}$ we used theory to guide the design of the SOLAS intervention, its delivery and the training of PTs in the feasibility trial. The current lack of theorydriven interventions in chronic pain populations ${ }^{38-40}$ may partly explain the poor uptake and continuation of SM behaviours, such as physical activity, while conversely, the application of theory to an intervention enables theoretically identified mechanisms of action (ie, mediators) to be investigated allowing greater understanding of how an intervention works and how it can be enhanced to improve outcomes. ${ }^{41-43}$ The SOLAS intervention is underpinned by self-determination theory, ${ }^{44}{ }^{45}$ which aims to increase patients' autonomous motivation and perceived competence to self-manage through the needs supportive environment created by the PT within the group as we have previously demonstrated in individual PT. ${ }^{25}{ }^{46}$ The intervention is also designed to target specific determinants of SM behaviour (knowledge, skills, self-efficacy/perceived competence, fear, catastrophising, motivation, behaviour regulation) in order to increase physical activity and the use of specific evidence-based SM behaviours (ie, pain management using pharmacological and nonpharmacological approaches, pain coping strategies, healthy eating for lifestyle and weight management and specific exercise) leading to changed health outcomes as outlined in figure 1 . As the potential for the SOLAS intervention to result in a change in the target SM behaviours is uncertain, the contributing assumptions of this underlying theoretical model of behaviour change will be explored within the feasibility trial ${ }^{47}$ and will further inform the design of a future effectiveness trial.

\section{Aims and objectives}

The aim of this cluster randomised controlled feasibility trial is to evaluate the feasibility and costs of providing 


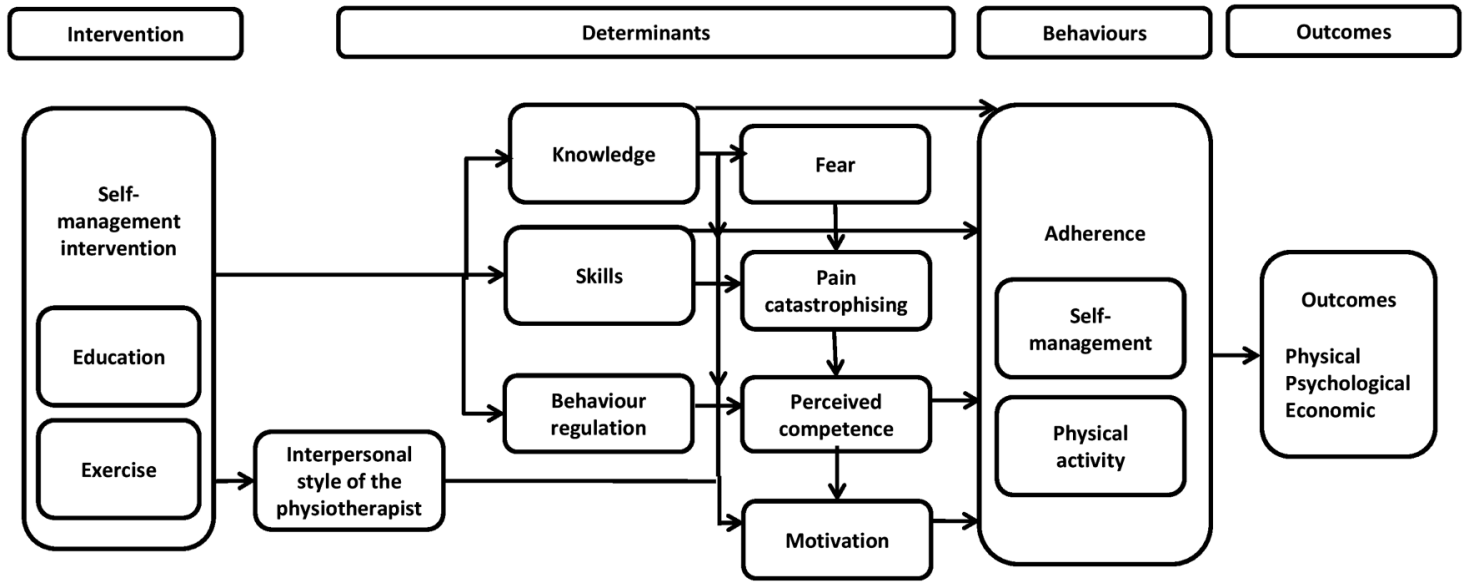

Figure 1 Process map of behaviour change in SOLAS intervention.

the SOLAS intervention (experimental group) to promote SM for patients with OA hip/knee and/or CLBP compared with usual PT, which will serve as the pragmatic control group in order to determine the feasibility of moving to a full scale trial by following the MRC guidelines. $^{31} 48$

Our primary objectives are: (1) to assess the acceptability and demand of the SOLAS intervention to patients and physiotherapists compared with usual PT in order to optimise its design, uptake and delivery; and (2) to determine the feasibility of trial procedures and the most efficient and effective study design for a definitive RCT, including its sample size. See table 1 below for details of feasibility aspects. The secondary objectives are: (3) to assess medium-term changes in physical function, pain, emotional and global well-being; (4) to evaluate contributing assumptions of the underlying theoretical model of behaviour change of the SOLAS intervention compared with usual PT; and (5) to determine the costs of the SOLAS intervention compared with usual PT from the health service and societal perspectives.

\section{METHODOLOGY}

\section{Design and setting}

An assessor-blinded multicentre two-arm non-inferiority cluster randomised controlled feasibility trial (allocation ratio 1:1) has been designed to assess the methodology proposed for use in a definitive RCT. Managers of nine publicly funded Health Service Executive (HSE) outpatient primary care PT areas in the greater Dublin, Ireland, area (population 1.25 million) have agreed to participate. Centres include 18 HSE primary community and continuing care clinics (hereafter referred to as PCCC clinics) with appropriate facilities, equipment and staffing. A full list of study sites is available from the principal investigator (PI). Eligible PTs will be purposively selected by PT managers based on affiliation with suitable study sites, interest, experience and caseload. Prior to randomisation, all identified PTs will receive a PT participant information leaflet, and invitation to provide written consent to confirm their interest by the project manager, which will involve completing training (intervention arm) and treatment of patients in the relevant trial arm. PTs will be invited to contact the project manager with any questions (see figure 2) and written informed consent will be obtained prior to their participation. PTs will not be blinded to treatment allocation, as they are implementing the intervention with HSE patients.

\section{Participants}

The following eligibility criteria will be applied to patients referred for PT to one of the participating PCCC clinics:

Inclusion: Patients will: have chronic ( $\geq 3$ months), $O A$ (National Institute for Health and Care Excellence (NICE; 2014) ${ }^{15}$ working diagnosis of OA hip/knee joint: age 45 years old or over, activity-related joint pain and no morning joint-related stiffness or morning stiffness that lasts $\leq 30 \mathrm{~min}$ ) and/or $L B P$ (age $\geq 30$ years old and have non-specific LBP of mechanical origin with or without radiation to the lower limb); be able to read/ understand and speak English without assistance; access to a telephone for screening and available to attend a 6-week group class of $1.5 \mathrm{~h} /$ week.

Exclusion: Patients will be excluded if: they have serious spinal pathology or previous spinal surgery, nerve root compromise, lower limb arthroplasty, systemic/inflammatory or cardiac disease, are scheduled for major surgery, confounding conditions (neurological, intellectual or unstable psychiatric condition), bladder/bowel incontinence, are assessed to be high risk of falls, had PT in preceding 6 months, are unable/ unwilling to attend or ongoing litigation related to their pain condition.

Identification, invitation, screening and recruitment Identifying eligible participants will be a multilevel process which has been established during the 


\begin{tabular}{|c|c|c|c|c|}
\hline \multirow[b]{3}{*}{ Feasibility } & \multicolumn{4}{|l|}{ Operational definitions } \\
\hline & \multicolumn{2}{|l|}{ SOLAS intervention } & \multicolumn{2}{|l|}{ Trial procedures } \\
\hline & Participants & Physiotherapists & Participants & Physiotherapists \\
\hline Acceptability & $\begin{array}{l}\text { The extent to which participants } \\
\text { who have received the SOLAS } \\
\text { intervention consider the content, } \\
\text { mode of delivery and support } \\
\text { materials acceptable, appropriate, } \\
\text { and satisfactory in meeting their } \\
\text { needs }\end{array}$ & $\begin{array}{l}\text { The extent to which physiotherapists } \\
\text { who have delivered the SOLAS } \\
\text { intervention consider the training, } \\
\text { content, mode of delivery and support } \\
\text { materials acceptable and appropriate in } \\
\text { meeting their needs and those of their } \\
\text { patients within their service context }\end{array}$ & $\begin{array}{l}\text { The extent to which participants } \\
\text { consider taking part in the trial, and } \\
\text { follow-ups, outcome measure } \\
\text { completion, and fidelity procedures } \\
\text { (direct observation of PTs delivering } \\
\text { intervention) acceptable and } \\
\text { appropriate }\end{array}$ & $\begin{array}{l}\text { The extent to which physiotherapists } \\
\text { who have participated in the trial } \\
\text { consider trial recruitment and fidelity } \\
\text { procedures acceptable and } \\
\text { appropriate }\end{array}$ \\
\hline Demand & $\begin{array}{l}\text { The extent to which participants } \\
\text { adhere to and perceive the burden } \\
\text { of SOLAS intervention weekly class } \\
\text { attendance and target behaviours }\end{array}$ & $\begin{array}{l}\text { The extent to which physiotherapists } \\
\text { perceive the demand and positive/ } \\
\text { negative effects of participating in } \\
\text { training, studying intervention } \\
\text { materials, preparing class venue and } \\
\text { delivering the SOLAS intervention, } \\
\text { using specified behaviour change } \\
\text { strategies }\end{array}$ & $\begin{array}{l}\text { The extent to which participants } \\
\text { perceive the burden of participating } \\
\text { in follow-up and completing specific } \\
\text { outcome measures within the trial }\end{array}$ & $\begin{array}{l}\text { The extent to which physiotherapists } \\
\text { perceive the demand of completing } \\
\text { their required tasks for participating in } \\
\text { the trial, including fidelity procedures } \\
\text { (self-report forms) }\end{array}$ \\
\hline Implementation & & $\begin{array}{l}\text { The quality and extent to which the } \\
\text { SOLAS intervention will be delivered } \\
\text { as planned by physiotherapists who } \\
\text { have completed training }\end{array}$ & & \\
\hline Practicality & & $\begin{array}{l}\text { The factors influencing the } \\
\text { implementation of the SOLAS } \\
\text { intervention in a range of HSE settings } \\
\text { by a range of physiotherapists taking } \\
\text { into account variations in staffing, } \\
\text { facilities, equipment and class size }\end{array}$ & & \\
\hline Adaptation & $\begin{array}{l}\text { The extent to which the SOLAS } \\
\text { intervention content, mode of } \\
\text { delivery and support materials will } \\
\text { need to be modified to enhance its } \\
\text { acceptability and implementation for } \\
\text { a future definitive trial }\end{array}$ & $\begin{array}{l}\text { The extent to which the SOLAS } \\
\text { intervention training, programme } \\
\text { content, mode of delivery and support } \\
\text { materials will need to be modified } \\
\text { during/at end of the trial to enhance its } \\
\text { acceptability and implementation for a } \\
\text { future definitive trial }\end{array}$ & $\begin{array}{l}\text { The extent to which trial recruitment, } \\
\text { follow-up and fidelity procedures } \\
\text { and the number and range of } \\
\text { outcome measures will need to be } \\
\text { modified during/at end of the trial to } \\
\text { enhance their acceptability and } \\
\text { implementation for a future definitive } \\
\text { trial }\end{array}$ & $\begin{array}{l}\text { The extent to which trial recruitment } \\
\text { and fidelity procedures, including } \\
\text { physiotherapists tasks, will need to be } \\
\text { modified during/at end of the trial to } \\
\text { enhance acceptability and } \\
\text { implementation for a future definitive } \\
\text { trial }\end{array}$ \\
\hline Integration & & $\begin{array}{l}\text { The perceived sustainability and level } \\
\text { of system change that will be needed } \\
\text { to integrate the SOLAS intervention } \\
\text { PT training and programme into } \\
\text { existing HSE community } \\
\text { physiotherapy services }\end{array}$ & & \\
\hline
\end{tabular}




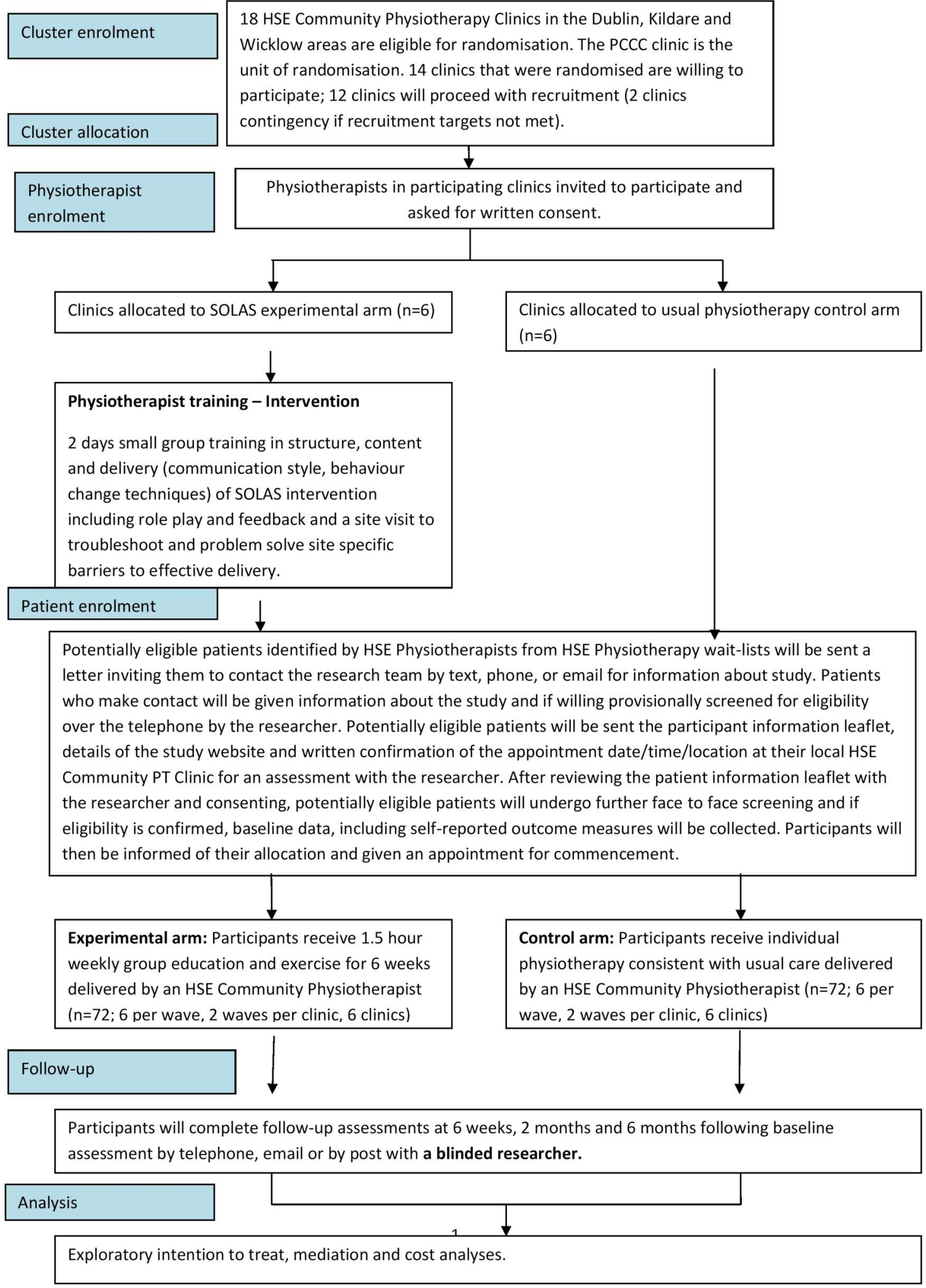

Figure 2 Study recruitment, allocation and follow-up (HSE, Health Service Executive; PCCC, primary community and continuing care; SOLAS, Self-management of Osteoarthritis and Low back pain through Activity and Skills).

development phase of this project in agreement with participating PCCC areas. It will involve the research team providing referring general practitioners' (GPs) with information about the trial, and intervention arms, PTs raising awareness of the trial at PCCC level, reviewing waiting lists for suitable referrals and sending potentially suitable patients an invitation letter, and the research team undertaking telephone and face-to-face screening and assessment of consenting participants as outlined in figure 2. 
All referrals to participating PCCC clinics will be reviewed for any potentially eligible patients with hip, knee or back pain by PTs (age, diagnosis, duration of symptoms), who will be sent standardised letters inviting them to contact the research team for information about the study by phoning (landline or trial mobile), texting or emailing as preferred. Those who make contact will be provided with information about the study, an opportunity to ask questions and if in agreement will be provisionally screened by the researcher (Chartered PT) via telephone using a standardised questionnaire (eligibility criteria, Physical Activity Readiness Questionnaire to confirm medical fitness to participate in the exercise class ${ }^{49}$ ) taking no longer than $10 \mathrm{~min}$. Interested and potentially eligible patients will be invited to attend their local PCCC clinic for face-to-face screening and assessment by the researcher. If indicated the GP will be contacted after screening or assessment to confirm the patient is suitable to participate. Patients will receive a letter containing the participant information leaflet, study website, confirmation of their appointment date/time/location, directions and contact details for queries and a reminder text $24 \mathrm{~h}$ in advance. The researcher will not be blinded to the allocation of each site but will not inform the patient of their randomisation allocation until completion of baseline assessment. A second blinded researcher will review the reasons for inclusion/exclusion of each patient to confirm lack of bias of the unblinded researcher.

At the face-to-face assessment, the participant information leaflet will be reviewed with the researcher and an opportunity provided to ask questions about the study and what participation involves, before providing written informed consent. Consenting participants will then proceed with screening and assessment, which will follow a standardised PT subjective and objective assessment for the relevant joint area, and balance testing if indicated as in other similar trials. ${ }^{50}$ If eligibility is confirmed, baseline data including the outcome measures will be completed with support from the researcher, who will read the questions to each participant unless he/she prefers to complete independently.

Participants will then be informed of their allocation by the researcher (based on the random allocation of PCCC clinic), the aim of the trial will be reiterated, and an appointment for the start will be provided. The researcher will notify PTs which patients have given informed consent and are eligible to participate. All other patients will be informed of the reason(s) for ineligiblity (if appropriate), will remain on the waiting list and advised that they will receive routine PT as soon as an appointment is available as per usual practice. Participants will be advised that they are free to withdraw from treatment and the study at any stage.

\section{Interventions}

The interventions are described below in accordance with current guidelines (SPIRIT, ${ }^{51}$ TIDieR $^{37}$ and
Borek ${ }^{52}$ ). Participants in both groups will be advised to continue their normal daily routines and medication, but will be requested to avoid any other treatment for their back, hip or knee pain during the study period.

\section{SOLAS intervention}

The overarching aim of the SOLAS intervention is to promote patient SM behaviour, that is, physical activity and specific SM strategies, through a group exercise and education class and accompanying support materials. See table 2 for details. The intervention is structured and multicomponent involving (1) education to increase knowledge about the individual's chronic condition, its consequences, and its SM including the role of a healthy lifestyle and available community resources and (2) training to impart increased SM behaviours at the end of the intervention and to enable participants to deploy these enhanced skills in their lives beyond the intervention. Participants randomised to this arm will receive six consecutive sessions in a group of up to six participants led by a PT within a PCCC clinic or nearby community facility. The overall aim of the programme and the importance of weekly attendance will be explained throughout to improve adherence. To support the promotion of participant SM behaviour, its determinants have been mapped to each session and relevant behaviour change techniques incorporated within sessions. In line with SDT and the importance of social agents in facilitating autonomous motivation and perceived competence for long-term behaviour change, the PT plays a crucial role by being needs supportive and enabling participants to SM by seeking their input, avoiding controlling language such as 'should' and 'must'; and trying to understand their perspective. The content and dose of each class (including attendance record, rates and reasons for non-attendance or early withdrawal of each participant) will be recorded by the treating therapist in a weekly treatment record form.

\section{Physiotherapist training in SOLAS intervention rationale, content and delivery}

Prior to implementation, PTs will complete a 2-day (that is, $12 \mathrm{~h}$ ) small group training course (up to $8 \mathrm{PTs}$ ), designed and co-facilitated by the intervention developers; a physiotherapist and senior researcher (DAH) who holds an MSc in musculoskeletal PT and a PhD in back pain research, and a registered psychologist and researcher (JM) who holds an MA in organisational and social psychology and a PhD in sport and exercise psychology. The course aims to introduce PTs to the SOLAS intervention structure, content, support materials and delivery using a needs supportive interpersonal style. Training incorporates active participation, collaboration and experiential learning, ${ }^{54}$ using multiple learning methods; brief PowerPoint presentations, videos of 'good ' and 'poor' intervention delivery, troubleshooting intervention implementation, and case-based role plays and microteaching activities to practice intervention delivery. 
Self-management behaviours targeted

Session within the session

\begin{tabular}{ll}
\hline 1 & i. Physical activity \\
iia. Specific exercise for pain condition
\end{tabular}

\section{Intervention content and participant materials}

Education: aims of programme, back pain and OA causes, cycle of change, exercise recommendations, physical activity levels in Ireland, benefits of exercise/physical activity, review of SOLAS exercise programme, physical activity diary and goal setting Exercise: explanation and demonstration of all exercise stations, practice of sample of exercises

Materials: participant programme handbook

\section{$2 \quad$ i. Physical activity}

iia. Specific exercise for pain condition

\section{$3 \quad$ i. Physical activity}

iia. Specific exercise for pain condition iib. Healthy eating for lifestyle and balanced weight

\section{Education: activity rest cycle and pacing activities, use of pedometer, walking technique, understanding pain, physical activity diary, goal setting and action plan \\ Exercise: supervised group exercise class \\ Materials: Yamax SW-200 Pedometer}

Education: balanced weight, obesity levels in Ireland, healthy eating, measuring waist circumference, physical activity diary, goal setting and action plan, food and drink diary

Exercise: supervised group exercise class

Materials: tape measure, Your Guide to Healthy Eating using the Food Pyramid, 101+Square Meals cookbook

Behaviour change techniques embedded within sessions (as pe Michie et al) ${ }^{53}$

1.1 Goal-setting [behaviour]

1.2 Problem -solving

1.3 Goal-setting (outcome)

1.4 Action planning

1.5 Review behaviour goal

1.6 Discrepancy between current

behaviour and goal

1.7 Review outcome goa

1.8 Behavioural contract

2.2 Feedback on behaviour

2.3 Self-monitoring of behaviour

2.7 Feedback on outcome of behaviour

3.1 Social support [unspecified]

3.2 Social support [practical]

3.3 Social support [emotional]

4.1 Instruction on how to perform the behaviour

4.2 Information about antecedents of the behaviour

5.1 Information about health consequences of the behaviour

5.6 Information about emotional consequences of the behaviour

6.1 Demonstration of the behaviour

$4 \quad$ i. Physical activity

iia. Specific exercise for pain condition iic. Use evidence-based pain management approaches to self-manage pain condition
Education: mid-way review, evidence-based pain management with ice/ heat, medication, TENS, acupuncture, physical activity diary, goal setting and action plan

Exercise: supervised group exercise class
6.2 Social comparison

8.1 Behavioural practice/rehearsal 8.6 Generalisation of target behaviour 8.7 Graded tasks

9.1 Credible source

10.4 Social reward

11.1 Pharmacological support
$5 \quad$ i. Physical activity

iia. Specific exercise for pain condition iid. Use of pain coping strategies
Education: anxiety, mood and pain, managing flare-ups, pain coping strategies, relaxation techniques and practice, physical activity diary, goal setting and action plan

Exercise: supervised group exercise class

Relaxation skills: practical group session of contract-relax relaxation technique led by PT Materials: relaxation CD
11.2 Reduce negative emotions 12.5 Adding objects to the environment 12.6 Body changes

13.2 Framing/Reframing

15.1 Verbal persuasion about capability 


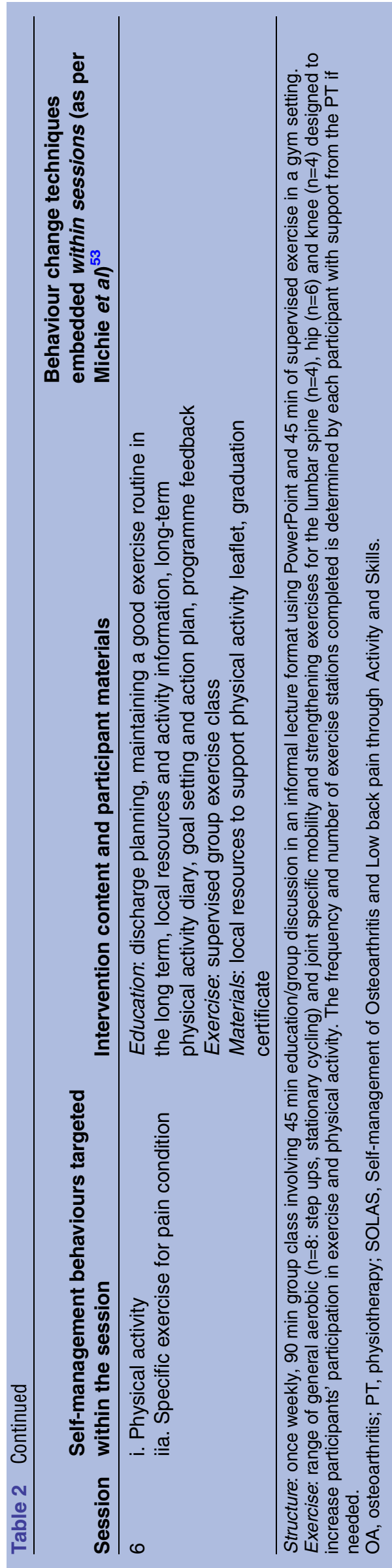

Each PT will receive precourse materials (ie, intervention handbook, slides and selected research papers) and following training additional intervention materials and individualised feedback from one of the course facilitators based on audio recordings during the course.

\section{Usual individual PT}

Participants in the control arm will receive usual individualised PT management, defined as usual PT treatment in primary care, which is generally in accordance with international evidence-based guidelines for $\mathrm{OA}$ and $\mathrm{CLBP}^{15}{ }^{55}$; advice on prescribed exercise, manual therapy and exercise therapeutic approaches, with advice and education regarding general lifestyle-related factors including physical activity and healthy diet less common. ${ }^{56}$ The content and dose (frequency and duration) of treatment provided to each participant will be at the discretion of the treating PT and explained to participants to promote adherence. The rate and reasons for non-attendance and/or early withdrawal will be documented by the treating PT in a standardised treatment record form. Recruitment will be from the top of the waiting list to minimise delay in starting treatment and to allow comparability at follow-up points with the experimental arm. PTs will not be permitted to refer participants to a group-based programme for pain management during the trial.

\section{Treatment fidelity}

A range of quantitative methods (ie, direct observation and audio recording by researcher, PT self-report) at the level of treating PTs in both study arms will be used on at least $20 \%$ of the treatment delivery data to assess the content and quality of treatment fidelity during the trial, in addition to qualitative interviews with intervention PTs to assess the feasibility of implementation and its practicality. ${ }^{57}$ Fidelity will be assessed and reported by separate evaluators from the outcome evaluators. The PI will be the integration point between the fidelity and outcome evaluation teams. ${ }^{48}$

\section{Randomisation and blinding}

The PCCC clinics will be the unit of randomisation (cluster) and each clinic will be randomised to the experimental (SOLAS intervention) or control (usual individual PT) arm on a 1:1 basis. In total, 18 suitable PCCC clinics initially identified as suitable for participation were randomised. Following review of geographical proximity of adjacent clinics, current availability of PT staff to participate and withdrawal of clinics, 14 randomised clinics were agreed with PT managers as available at the start of the trial. Twelve clinics will proceed with recruitment (six experimental and six control) and two clinics (one experimental and one control) will be held as contingency in case recruitment targets are not met. Randomisation will be conducted using a computerised random number generator algorithm by a statistician blinded to the purpose of 
Table 3 Feasibility and process of behaviour change outcomes and measures

\begin{tabular}{|c|c|c|c|c|}
\hline Variable & Measure and items & Details & $\begin{array}{l}\text { Reliability where } \\
\text { available }\end{array}$ & $\begin{array}{l}\text { Administration } \\
\text { point, trial arms }\end{array}$ \\
\hline Expectation of treatment & $\begin{array}{l}\text { Expectation of treatment } \\
\text { scale }^{58} \\
\text { 4-items }\end{array}$ & $\begin{array}{l}\text { Patients and PTs rate how helpful they believe both } \\
\text { individual and group treatment will be for OA and CLBP. } \\
\text { Measured with 10-point numeric rating scales ranging from } \\
\text { not at all helpful to extremely helpful }\end{array}$ & $\begin{array}{l}\text { Internal } \\
\text { consistency: } 0.84^{58}\end{array}$ & Baseline only \\
\hline $\begin{array}{l}\text { Client satisfaction with outcome } \\
\text { and care }\end{array}$ & $\begin{array}{l}\text { Satisfaction } \\
\text { questionnaire } \\
2 \text {-items }\end{array}$ & $\begin{array}{l}\text { Patients rate their satisfaction with PT care and their feelings } \\
\text { on hypothetically living the rest of their life with current } \\
\text { symptoms. Measured using 5-point numeric rating scales }\end{array}$ & $\begin{array}{l}\text { Internal } \\
\text { consistency: } \\
0.87-0.90^{60}\end{array}$ & 2 months, 6 months \\
\hline $\begin{array}{l}\text { Acceptability of treatment and trial } \\
\text { participation }\end{array}$ & $\begin{array}{l}\text { Brief questionnaire } \\
\text { developed for this trial } \\
11 \text {-items }\end{array}$ & $\begin{array}{l}\text { Patients rate their acceptability of treatment received and } \\
\text { trial participation, including the burden of outcome measure } \\
\text { completion. Measured using 5-point numeric rating scales } \\
\text { and yes/no responses }\end{array}$ & $\begin{array}{l}\text { No reliability data } \\
\text { available }\end{array}$ & 6 months \\
\hline Physical activity & $\begin{array}{l}\text { International Physical } \\
\text { Activity Questionnaire } \\
\text { 7-items }\end{array}$ & $\begin{array}{l}\text { Patients provide time spent undertaking vigorous/moderate } \\
\text { physical activity, walking and being sedentary in the last } \\
7 \text { days }\end{array}$ & $\begin{array}{l}\text { Test-retest reliability } \\
\text { range: } 0.46-0.96^{61}\end{array}$ & $\begin{array}{l}\text { Baseline, week } 6 \text {, } \\
2 \text { months, } 6 \text { months }\end{array}$ \\
\hline $\begin{array}{l}\text { SOLAS self-management } \\
\text { behaviours }\end{array}$ & $\begin{array}{l}\text { Brief questionnaire } \\
\text { developed for this trial* } \\
6 \text {-items }\end{array}$ & $\begin{array}{l}\text { Patients describe adherence to target self-management } \\
\text { behaviours in the past week on yes/no and number of day } \\
\text { scales }\end{array}$ & $\begin{array}{l}\text { No reliability data } \\
\text { available }\end{array}$ & $\begin{array}{l}\text { Baseline, week } 6 \text {, } \\
2 \text { months, } 6 \text { months }\end{array}$ \\
\hline Pain catastrophising & $\begin{array}{l}\text { Pain catastrophising } \\
\text { scale }^{62} \\
13 \text {-items }\end{array}$ & $\begin{array}{l}\text { Patients indicate their agreement with items following the } \\
\text { question 'when in pain' such as I anxiously want the pain to } \\
\text { go away' on 5-point scales ranging from 'not at all' to 'all of } \\
\text { the time' }\end{array}$ & $\begin{array}{l}\text { Internal } \\
\text { consistency: } 0.95^{63}\end{array}$ & $\begin{array}{l}\text { Baseline, week } 6 \text {, } \\
2 \text { months, } 6 \text { months }\end{array}$ \\
\hline Fear & $\begin{array}{l}\text { Tampa Scale of } \\
\text { Kinesiophobia }^{64} \\
11 \text {-items }\end{array}$ & $\begin{array}{l}\text { Patient rate beliefs about their pain on a 4-point scale } \\
\text { ranging from strongly disagree to strongly agree }\end{array}$ & $\begin{array}{l}\text { Internal } \\
\text { consistency: } 0.91^{65}\end{array}$ & $\begin{array}{l}\text { Baseline, week } 6 \text {, } \\
2 \text { months, } 6 \text { months }\end{array}$ \\
\hline Self-efficacy & $\begin{array}{l}\text { Pain Self-Efficacy } \\
\text { Questionnaire } \\
\text { 10-items }\end{array}$ & $\begin{array}{l}\text { Patients rate confidence in performing various activities with } \\
\text { their pain on a 7-point scale ranging from not at all confident } \\
\text { to completely confident }\end{array}$ & $\begin{array}{l}\text { Internal } \\
\text { consistency: } 0.92^{66}\end{array}$ & $\begin{array}{l}\text { Baseline, week } 6 \text {, } \\
2 \text { months, } 6 \text { months }\end{array}$ \\
\hline Autonomy support from PT & $\begin{array}{l}\text { Health Care Climate } \\
\text { Questionnaire } \\
\text { 15-items }\end{array}$ & $\begin{array}{l}\text { Patients rate aspects of their interaction with their PT during } \\
\text { treatment, such as 'I feel understood by my physiotherapist' } \\
\text { on a 7-point scale ranging from strongly disagree to strongly } \\
\text { agree }\end{array}$ & $\begin{array}{l}\text { Internal } \\
\text { consistency: } 0.96^{67}\end{array}$ & Week 1, week 6 \\
\hline $\begin{array}{l}\text { Autonomous and controlled } \\
\text { motivation (to follow PTs advice } \\
\text { to self-manage) }\end{array}$ & $\begin{array}{l}\text { Treatment } \\
\text { Self-Regulation } \\
\text { Questionnaire } \\
\text { 9-items }\end{array}$ & $\begin{array}{l}\text { Patients rate their agreement with statements on why they } \\
\text { follow their PT's advice on a 7-point scale ranging from not } \\
\text { true for me to very true for me }\end{array}$ & $\begin{array}{l}\text { Internal } \\
\text { consistency: } 0.76^{68}\end{array}$ & $\begin{array}{l}\text { Baseline, week } 6 \text {, } \\
2 \text { months, } 6 \text { months }\end{array}$ \\
\hline
\end{tabular}

Continued 


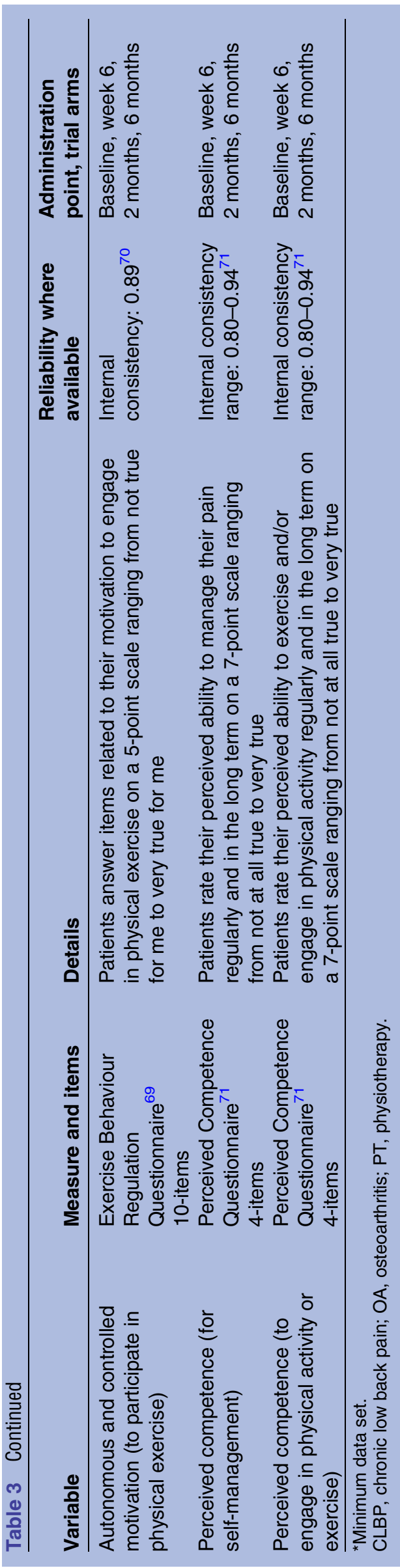

the study. A researcher will contact all PT managers to inform them of their allocation arm.

Participant and clinician blinding is not possible due to the nature of the intervention, and it is also not possible to blind the researcher at baseline due to the cluster nature of the study. All follow-up outcome assessments will be conducted by a blinded assessor member of the research team. The investigators responsible for data analysis will use a coded data set to ensure blinding. Semistructured interviews, which include treatmentspecific questions, will be conducted with intervention PTs at the end of delivery, and with participants after the end of outcome measure data collection by unblinded researchers.

\section{Outcomes and measures}

\section{Primary outcomes}

The primary outcomes are related to the feasibility of the SOLAS intervention and trial design and procedures. For participants, this will be assessed by questionnaires (see table 3 below) of treatment expectation, satisfaction, acceptability and demand of treatment and trial participation, as well as attendance rates and individual qualitative interviews described below. For physiotherapists, a range of feasibility aspects will be assessed by expectation of treatment questionnaires, individual and focus group qualitative studies. Additionally, the feasibility of trial design and procedures will be assessed by piloting the methodological procedures, monitoring the recruitment and retention rates and procedures, number and reasons for withdrawal during the treatment process, feasibility of outcome measurement, follow-up and fidelity procedures, refining the training programme and calculating the sample size for a definitive trial by estimating the effect sizes and intracluster correlation coefficients of secondary outcomes.

\section{Secondary measures}

At baseline, demographic data including age, gender, occupational status and clinical presentation will be documented as well as specific factors that could influence the treatment effect; that is, therapists' personality (causality orientations), motivation to participate and treatment expectations; participants' level of risk of chronicity (CLBP participants only). The selected secondary outcome measures are core outcomes for chronic pain trials including physical functioning, pain intensity, pain bothersomeness, emotional functioning, quality of life and global ratings of improvement, ${ }^{72-74}$ as well as recommended economic outcomes. ${ }^{75}$ The process of change in selected mediators (pain catastrophising, fear, self-efficacy/perceived competence and motivation) and engagement in SM behaviours (physical activity and specific SM strategies) will be assessed using a range of measures. The cost of the SOLAS intervention compared with individual PT will be evaluated using several resource utilisation and health state measures. 
See table 3 and online supplementary file 2 for details of all measures.

\section{Data collection}

Secondary outcomes will be collected at baseline, 2 and 6 months from baseline/start of treatment, and there will be an additional 6 -week follow-up time point for collection of process of behaviour change outcomes using the same data collection methods. This will involve a face-to-face interview with a researcher at baseline, and completion at all follow-up points with a blinded researcher by telephone to minimise participant data entry errors. The time for completion will be documented. Participants will receive an information letter and questionnaire pack 1 week prior to each follow-up point, and telephone contact to arrange a convenient time for completion with the researcher. Participants will have the option to complete outcome assessment by post if preferred. Non-respondents will be contacted by phone/text message on three occasions within a 3-day period to invite completion of outcomes, and if no response is obtained will be posted the outcome measure pack (minimum data set) and prepaid envelope, and receive a text reminder inviting completion at their convenience. All researchers will receive training in interview skills and outcome measure administration by the PI prior to the start.

\section{Qualitative studies}

Individual qualitative semistructured telephone interviews will be conducted with a purposive sample of consenting participants who received the SOLAS intervention or usual PT (2:1 ratio) on completion of the 6-month follow-up to explore in depth their experience of treatment and trial participation, necessary adaptations to optimise acceptability and uptake, and their use of target SM behaviours. Individual qualitative semistructured telephone interviews will be conducted with all PTs following delivery of the SOLAS intervention to establish their views on the acceptability and demand of the training course, SOLAS intervention, trial recruitment and fidelity procedures and necessary adaptations to optimise acceptability and implementation. On completion of the trial, a focus group with PT managers and a purposive sample of PTs will assess views on the practicality and potential future integration of the SOLAS intervention into existing PCCG services to further inform a definitive trial.

\section{Sample size}

We plan to recruit between 12 and 14 clusters (PCCC sites) to ensure the intervention is feasible across a range of settings with variable staffing, facilities, equipment and clientele. There will be a minimum of six clusters in each arm, participating in two waves of recruitment with the aim to recruit six participants in each cluster per wave, resulting in 144 participants (72 per arm). Additional clusters $(n=2)$ will only be included as a contingency if recruitment targets are not met and added to the final study waves. Recommendations for feasibility studies suggest that the data set for analysis include at least 30 participants per arm in order to estimate parameters for future sample size calculations. ${ }^{76-78}$ Accounting for the cluster design effect and assuming an ICC coefficient of $0.03,{ }^{79}$ an effective sample size of 30 would require 36 participants per arm. Allowing for $25 \%$ loss to follow-up, we would need to recruit 48 participants per arm (96 in total). Therefore, this study size will be sufficiently large to allow a precise estimate of the ICC coefficient; the greater the number of clusters the more precise the estimate of the ICC coefficient. ${ }^{80}$ This study design and sample size should therefore satisfy the dual aims of (1) feasibility and (2) estimation of statistics to enable a future sample size calculation for a definitive trial.

\section{Data integrity}

All hard copy and audio-recorded data will be kept confidential and stored in a locked filing cabinet in the research group office and in softcopy in a passwordprotected database only accessible to the PI, data manager and statistician. The research team will monitor the integrity of trial data. All participant, group allocation, treatment record and sociodemographic data will be coded, and outcome questionnaires scored, and all data will be double entered into the Statistical Package for the Social Sciences package to detect and correct input errors, and range checks will be undertaken prior to data analysis. A research investigator unblinded to treatment allocation will perform regular data checks during data entry and provide feedback to PTs regarding data omissions where necessary.

\section{Planned analyses}

Since this is a feasibility study, extensive exploratory and descriptive analysis of the data will be undertaken. A combination of quantitative and qualitative methods will be used to answer the primary research objectives related to the feasibility of the intervention and trial procedures to participants and PTs to determine the feasibility and most efficient and effective study design for a definitive trial. Statistical analysis will be by intention to treat according to participants' assigned arm of the study, regardless of whether they attend for treatment or not. The data analysis plan for treatment fidelity will be reported separately.

Analysis of the feasibility aspects will be undertaken on an interim basis after each study wave by the UCD study team and used to inform minor protocol refinements for subsequent waves. Qualitative data will be analysed using inductive thematic analysis, based on Braun and Clarke's $^{81}$ method. Coding frames will be developed from a review of provisional themes, which will then be re-examined and refined. The reliability of the identified themes will be established by a second researcher who will independently code a random sample of $25 \%$ of 
each data set using the coding frame, with $70 \%$ agreement taken as the minimum cut-off rate of agreement. ${ }^{82}$ The recruitment rate overall, and according to study arm and PCCC clinic will be calculated in addition to the ratio of number screened: number enrolled. The conversion rate and reasons for exclusion/refusal/withdrawal at each stage in the recruitment process and the attrition rate during the treatment period will be evaluated. The feasibility of outcome measurement procedures will be assessed by the time to complete measures, the number of missing items according to outcome measure, and the follow-up rates and methods of completion (phone, post) at each time point and participants' reported burden of completion.

Analysis of the secondary outcome measure data will be undertaken at the end of the trial and performed by the statistician who will remain blinded to group identification until analysis is complete. A linear mixed model will be used to examine change over time in participant outcomes between treatment groups, while adjusting for study waves and clinics, and potentially individual PTs. Treatment effects will be reported with $95 \%$ CIs and ICCs for the clusters. The results will guide a sample size calculation and the form of the primary analysis for a definitive trial. The relationships between the SOLAS intervention, PT autonomy support and the physical activity/SM behaviour measures, via potential mediators (pain catastrophising, fear, self-efficacy/perceived competence and motivation) will be explored, and contingent on adequate sample size, with a series of linear models, ${ }^{83}$ and by applying the test of Sobel,${ }^{84}$ and structural equation models, and extended to examine impact on secondary outcomes. Finally, the cost of the SOLAS intervention compared with usual PT will be calculated from the health service and societal perspectives using data from resource utilisation measures, alongside exploratory analysis of quality-adjusted life years (EQ-5D) and disease-specific measures. ${ }^{85}$

\section{Adverse effects or events}

No adverse events, apart from minor musculoskeletal complaints in the SOLAS intervention arm due to unaccustomed exercise, are anticipated. Treatment will be modified or discontinued if necessary due to an increase in symptoms. Participants and PTs will be informed that any adverse events should be reported immediately to the PI and will be documented by the research team by type, length of time and frequency should they occur.

\section{Trial governance}

The Trial Management Committee (TMC), comprising the PI and local co-investigators, provides overall management of the feasibility trial including PCCC clinic set-up, PT training, recruitment and screening procedures, trial promotion, data collection, analysis and interpretation. Trial conduct will be audited on a weekly basis using a standardised proforma during each recruitment wave, and biannually at the end of each recruitment wave. Members of the international scientific team provide independent and scientific oversight of the progress of the study through the Data Monitoring Committee on behalf of the funder (Health Research Board).

\section{ETHICS AND DISSEMINATION}

All patients and physiotherapists will be provided with detailed written information and have an opportunity to discuss participation before providing written consent and will be advised they can withdraw at any point. Patients will be invited to give their consent to participate in a telephone interview following completion of all follow-ups. The feasibility results will be published in peer-reviewed journals and presented at national and international academic, clinical and health service conferences. Any protocol amendments including changes to eligibility criteria, outcomes or analyses will be communicated to the UCD Human Research EthicsSciences Committee and the HSE Primary Care Research Committee, and noted on the Current Controlled Trials trial registration page and reported in the trial results papers.

\section{DISCUSSION}

The provision of SM healthcare interventions with robust evidence of clinical and cost-effectiveness for chronic conditions, including LBP and OA, are becoming increasingly important due to their rising prevalence and significant impact on the individual, their family and carers, employers and wider society. The complex multifaceted nature of supporting patients to engage in SM behaviours requires an underlying model of behaviour change to enhance the likelihood of long-term adherence and to understand the underlying processes of change. Group-based interventions that are first designed, adapted and tested for feasibility and cost implications in partnership with patients' and healthcare providers within local health service settings before robust evaluation in an effectiveness trial have the potential to address this second translational gap. The feasibility of the SOLAS theory-driven, group-based complex intervention will be evaluated from the patient, practitioner and health service manager levels, and if achieved the trial design will be refined based on the findings of this study before moving to a definitive trial.

\section{Author affiliations}

${ }^{1}$ Institute for Sport and Health and UCD School of Public Health, Physiotherapy and Sports Science, University College Dublin, Dublin, Ireland ${ }^{2}$ The George Institute for Global Health, Oxford Martin School, Oxford University, Oxford, UK

${ }^{3}$ Breast-Predict-Collaborative Cancer Research Centre, Pharmacology and Therapeutics, Trinity College Dublin, St James's Hospital, Dublin, Ireland ${ }^{4}$ Department of Psychology, University of London, Royal Holloway, London, UK

${ }^{5}$ The George Institute for Global Health, Sydney Medical School, University of Sydney, Sydney, New South Wales, Australia 
${ }^{6}$ Institute of Nursing and Health Research, Ulster University, Newtownabbey, UK

${ }^{7}$ Institute for Positive Psychology and Education, Faculty of Health Sciences, Australian Catholic University, New South Wales, Australia

${ }^{8}$ Faculty of Health and Applied Sciences, University of the West of England, Bristol, UK

${ }^{9}$ UCD School of Psychology, University College Dublin, Dublin, Ireland ${ }^{10}$ CSTAR and UCD School of Public Health, Physiotherapy and Sports Science, University College Dublin, Dublin, Ireland

Acknowledgements The authors wish to thank Collaborators from the SOLAS Trial team Professor Charles Normand and Dr James F O'Mahony for advice on the cost analysis, Marian Hernon for contribution to cost methods, and Alison Keogh and Elaine Toomey for contribution to fidelity methods. The authors also wish to thank William Fox and Isabelle Jeffares for assistance with manuscript preparation.

Collaborators Professor Charles Normand, Dr James F O'Mahony, Marian Hernon, Alison Keogh, Elaine Toomey.

Contributors DAH and AMH conceived the idea for the study and with input from TP, CM, SMM, SG, CL and SK secured funding. DAH and JM developed the SOLAS intervention and training programme with input from LC-M, AMH, SG and NEW. DAH and LC-M designed the trial with input from JM, AMH, RS, NEW, SG, SMM, CL, SK, CM and TP. RS, DAH and SG devised the planned analysis. DAH drafted the manuscript with critical input from all authors.

Funding This article presents independent research funded by the Health Research Board (Health Research Award 2012/24).

Competing interests None declared.

Ethics approval UCD Human Research Ethics-Sciences Committee, REC Reference: LS-13-54 Currie-Hurley. Research access: HSE Primary Care Research Committee.

Provenance and peer review Not commissioned; externally peer reviewed.

Open Access This is an Open Access article distributed in accordance with the Creative Commons Attribution Non Commercial (CC BY-NC 4.0) license which permits others to distribute, remix, adapt, build upon this work noncommercially, and license their derivative works on different terms, provided the original work is properly cited and the use is non-commercial. See: http:// creativecommons.org/licenses/by-nc/4.0/

\section{REFERENCES}

1. Vos T, Barber RM, Bell B, et al. Global, regional, and national incidence, prevalence, and years lived with disability for 301 acute and chronic diseases and injuries in 188 countries, 1990-2013: a systematic analysis for the Global Burden of Disease Study 2013. Lancet 2015;386:743-800

2. Cross M, Smith E, Hoy D, et al. The global burden of hip and knee osteoarthritis: estimates from the global burden of disease 2010 study. Ann Rheum Dis 2014;73:1323-30

3. March L, Smith EUR, Hoy DG, et al. Burden of disability due to musculoskeletal (MSK) disorders. Best Pract Res Clin Rheumatol 2014;28:353-66.

4. Ipsos MRBI Healthy Ireland Survey 2015. Dublin: The Stationery Office, 2015.

5. Institute of Public Health. Musculoskeletal conditions briefing. Dublin, 2012.

6. French HP, Galvin R, Horgan NF, et al. Prevalence and burden of osteoarthritis amongst older people in Ireland: findings from the Irish LongituDinal Study on Ageing (TILDA). Eur J Public Health 2015 [Published Online First: 23 June 2015].

7. Speerin $\mathrm{R}$, Slater $\mathrm{H}$, Li L, et al. Moving from evidence to practice: models of care for the prevention and management of musculoskeletal conditions. Best Pract Res Clin Rheumatol 2014;28:479-515

8. Musculoskeletal Health in Europe Report v5.0 Final. eumusc.net Driving musculoskeletal health for Europe, Executive Agency for Health and Consumers.

9. Slobbe L, Kommer G, Smit J, et al. Musculoskeletal Health in Europe Report v5.0 Final. eumusc.net Driving musculoskeletal health for Europe, Executive Agency for Health and Consumers.
Costs of illness in the Netherlands 2003. Bilthoven: Rijksinstituut voor Volksgezondheid en Milieu, 2006.

10. Raftery MN, Ryan P, Normand C, et al. The economic cost of chronic noncancer pain in Ireland: results from the PRIME study, part 2. Pain 2012;13:139-45

11. Wilkie R, Hay EM, Croft $P$, et al. Exploring how pain leads to productivity loss in primary care consulters for osteoarthritis: a prospective cohort study. PLOS ONE 2015;10:e0120042.

12. Buchbinder R, Blyth FM, March LM, et al. Placing the global burden of low back pain in context. Best Pract Res Clin Rheumatol 2013;27:575-89.

13. Fernandes L, Hagen KB, Bijlsma JW, et al. EULAR recommendations for the non-pharmacological core management of hip and knee osteoarthritis. Ann Rheum Dis 2013;72 1125-35.

14. McAlindon T, Bannuru R, Sullivan M, et al. OARSI guidelines for the non-surgical management of knee osteoarthritis. Osteoarthritis Cartilage 2014;22:363-88.

15. Excellence NIFHAC. Osteoarthritis. Care and management in adults Clinical Guideline 177. London: NICE, 2014.

16. Delitto A, George SZ, Van Dillen LR, et al. Low back pain. J Orthop Sports Phys Ther 2012;42:A1-57.

17. Savigny $P$, Watson $P$, Underwood $M$. Early management of persistent non-specific low back pain: summary of NICE guidance. BMJ 2009;338:b1805

18. Health Do. Supporting people with long term conditions: an NHS and social care model to support local innovation and integration. Leeds: Department of Health, 2005.

19. HSE. Framework for self-management support, long-term health conditions: HSE National Advocacy Unit. Secondary framework for self-management support, long-term health conditions. Dublin: HSE National Advocacy Unit, 2012.

20. Imison C, Naylor C, Buck D, et al. Transforming our healthcare system. London: The King's Fund, 2011.

21. Kroon FP, van der Burg LR, Buchbinder R, et al. Self-management education programmes for osteoarthritis. Cochrane Database Syst Rev 2014;1:CD008963.

22. Taylor SJ, Pinnock H, Epiphaniou E, et al. A rapid synthesis of the evidence on interventions supporting self-management for people with long-term conditions: PRISMS-practical systematic review of self-management support for long-term conditions. Southampton: NIHR Journals Library, 2014.

23. Carnes D, Homer K, Miles C, et al. Effective delivery styles and content for self-management interventions for chronic musculoskeletal pain: a systematic literature review. Clin J Pain 2012;28:344-54.

24. French HP, Cusack T, Brennan A, et al. Exercise and manual physiotherapy arthritis research trial (EMPART) for osteoarthritis of the hip: a multicenter randomized controlled trial. Arch Phys Med Rehab 2013;94:302-14.

25. Murray A, Hall A, Williams G, et al. Effect of a self-determination theory-based communication skills training program on physiotherapists psychological support for their patients with chronic low back pain: a randomized controlled trial. Arch Phys Med Rehab 2015;96:809-16.

26. McMahon S, Cusack T, O'Donoghue G. Barriers and facilitators to providing undergraduate physiotherapy clinical education in the primary care setting: a three-round Delphi study. Physiotherapy 2014:100:14-19.

27. Toomey EC, Currie-Murphy L, Matthews J, et al. The effectiveness of physiotherapist-delivered group education and exercise interventions to promote self-management for people with osteoarthritis and chronic low back pain: a rapid review part I. Man Ther 2015;20:365-86.

28. O'Farrell S, Smart K, Caffrey A, et al. Orthopaedic triage at a physiotherapist-led 'Musculoskeletal Assessment Clinic': a seven-month service evaluation of outcomes. Ir J Med Sci 2014:183:565-71.

29. Roddy E, Zwierska I, Jordan KP, et al. Musculoskeletal clinical assessment and treatment services at the primary-secondary care interface: an observational study. Br J Gen Pract 2013;63:e141-8.

30. Pasquale MK, Dufour R, Joshi AV, et al. Inefficiencies in osteoarthritis and chronic low back pain management. Am J Manag Care 2013;19:816-23.

31. Craig $P$, Dieppe $P$, Macintyre $S$, et al. Developing and evaluating complex interventions: the new Medical Research Council guidance. BMJ 2008;337:a1655.

32. Greaves CJ, Campbell JL. Supporting self-care in general practice. Br J Gen Pract 2007;57:814-21.

33. Hoddinott $\mathrm{P}$, Allan $\mathrm{K}$, Avenell $\mathrm{A}$, et al. Group interventions to improve health outcomes: a framework for their design and delivery. BMC Public Health 2010;10:800. 
34. Craig P, Petticrew M. Developing and evaluating complex interventions: reflections on the 2008 MRC guidance. Int J Nurs Stud 2013;50:585-7.

35. Bowen D, Kreuter M, Spring B, et al. How we design feasibility studies. Am J Prev Med 2009;36:452-7.

36. Campbell M, Fitzpatrick R, Haines A, et al. Framework for design and evaluation of complex interventions to improve health. $B M J$ 2000;321:694-6.

37. Hoffmann TC, Glasziou PP, Boutron I, et al. Better reporting of interventions: template for intervention description and replication TIDieR checklist and guide. BMJ 2014;348:g1687.

38. Williams A, Eccleston C, Morley S. Psychological therapies for the management of chronic pain (excluding headache) in adults. Cochrane Database Syst Rev 2012;11:CD007407.

39. Keogh A, Tully MA, Matthews J, et al. A review of behaviour change theories and techniques used in group based self-management programmes for chronic low back pain and arthritis. Man Ther 2015;20:727-35.

40. Marley J, Tully M, Poter-Armstrong A, et al. A systematic review of interventions aimed at increasing physical activity in adults with chronic musculoskeletal pain—protocol. Syst Rev 2014;3:106

41. Michie S, Abraham C. Identifying techniques that promote health behaviour change: Evidence based or evidence inspired? Psychol Health 2004;19:29-49.

42. Rothman AJ. "Is there nothing more practical than a good theory?": Why innovations and advances in health behavior change will arise if interventions are used to test and refine theory. Int $J$ Behav Nutr Phys Act 2004;1:11.

43. Rothman AJ. Capitalizing on opportunities to refine health behavior theories. Health Educ Behav 2009;36(5 Suppl):150S-5S; discussion 67S-71S.

44. Ryan D, Deci E. Self-determination theory and the facilitation of intrinsic motivation, social development and well being. Am Psychol 2000;55:68-78.

45. $\mathrm{Ng} J \mathrm{Y}$, Ntoumanis $\mathrm{N}$, Thøgersen-Ntoumani $\mathrm{C}$, et al. Self-determination theory applied to health contexts a meta-analysis. Perspect Psychol Sci 2012;7:325-40.

46. Matthews J, Hall AM, Hernon M, et al. A brief report on the development of a theoretically-grounded intervention to promote patient autonomy and self-management of physiotherapy patients: face validity and feasibility of implementation. BMC Health Serv Res 2015;15:260.

47. Courneya KS. Efficacy, effectiveness, and behavior change trials in exercise research. Int J Behav Nutr Phys Act 2010;7:81.

48. Moore GF, Audrey S, Barker M, et al. Process evaluation of complex interventions: Medical Research Council guidance. BMJ 2015;350: h1258.

49. Adams R. Revised physical activity readiness questionnaire. Can Fam Physician 1999;45:1004-5.

50. Walsh N, Cramp F, Palmer S, et al. Exercise and self-management for people with chronic knee, hip or lower back pain: a cluster randomised controlled trial of clinical and cost-effectiveness. Study protocol. Physiotherapy 2013;99:352-7.

51. Chan AW, Tetzlaff JM, Gotzsche PC, et al. SPIRIT 2013 explanation and elaboration: guidance for protocols of clinical trials. $B M J$ 2013;346:e7586

52. Borek AJ, Abraham C, Smith JR, et al. A checklist to improve reporting of group-based behaviour-change interventions. BMC Public Health 2015;15:963.

53. Michie S, Richardson M, Johnston M, et al. The behavior change technique taxonomy (v1) of 93 hierarchically clustered techniques: building an international consensus for the reporting of behavior change interventions. Ann Behav Med 2013;46:81-95.

54. Aelterman N, Vansteenkiste M, Keer HV, et al. Development and evaluation of a training on needs-supportive teaching in physical education: qualitative and quantitative findings. $J$ Teach Educ 2013;29:64-75.

55. Koes BW, van Tulder M, Lin CW, et al. An updated overview of clinical guidelines for the management of non-specific low back pain in primary care. Eur Spine J 2010;19:2075-94.

56. O'Donoghue G, Cunningham C, Murphy F, et al. Assessment and management of risk factors for the prevention of lifestyle-related disease: a cross-sectional survey of current activities, barriers and perceived training needs of primary care physiotherapists in the Republic of Ireland. Physiotherapy 2014;100:116-22.

57. Borelli B. The assessment, monitoring and enhancement of treatment fidelity in public health clinical trials. J Public Health Dent 2011;71:S52-63.

58. Smeets RJ, Beelen S, Goossens ME, et al. Treatment expectancy and credibility are associated with the outcome of both physical and cognitive-behavioral treatment in chronic low back pain. Clin J Pain 2008;24:305-15.
59. Hurley DA, Tully MA, Lonsdale C, et al. Supervised walking in comparison with fitness training for chronic back pain in physiotherapy: results of the SWIFT single-blinded randomized controlled trial (ISRCTN17592092). Pain 2015;156:131-47.

60. Fischer D, Stewart AL, Bloch DA, et al. Capturing the patient's view of change as a clinical outcome measure. JAMA 1999;282:1157-62.

61. Craig CL, Marshall AL, Sjöström M, et al. International physical activity questionnaire: 12 -country reliability and validity. Med Sci Sports Exerc 2003;35:1381-95.

62. Sullivan MJ, Bishop SR, Pivik J. The pain catastrophizing scale: development and validation. Psychol Assess 1995;7:524-532.

63. Osman A, Barrios FX, Gutierrez PM, et al. The pain catastrophizing scale: further psychometric evaluation with adult samples. J Behav Med 2000;23:351-65.

64. Kori SH, Miller RP, Todd DD. Kinesiophobia: a new view of chronic pain behavior. Pain Manag 1990;3:35-43.

65. Lundberg MK, Styf J, Carlsson SG. A psychometric evaluation of the Tampa Scale for Kinesiophobia-from a physiotherapeutic perspective. Physiother Theory Pract 2004;20:121-33.

66. Nicholas MK. The pain self-efficacy questionnaire: taking pain into account. Eur J Pain 2007;11:153-63.

67. Williams GC, Freedman ZR, Deci EL. Supporting autonomy to motivate patients with diabetes for glucose control. Diabetes Care 1998;21:1644-51.

68. Levesque CS, Williams GC, Elliot D, et al. Validating the theoretical structure of the treatment self-regulation questionnaire (TSRQ) across three different health behaviors. Health Educ Res 2007;22:691-702.

69. Mullan E, Markland D, Ingledew DK. A graded conceptualisation of self-determination in the regulation of exercise behaviour: development of a measure using confirmatory factor analytic procedures. Pers Individual Differences 1997;23:745-52.

70. Murcia JA, Gimeno EC, Camacho AM. Measuring self-determination motivation in a physical fitness setting: validation of the Behavioral Regulation in Exercise Questionnaire-2 (BREQ-2) in a Spanish sample. J Sports Med Phys Fitness 2007;47:366-74.

71. Williams GC, Deci EL. Internalization of biopsychosocial values by medical students: a test of self-determination theory. J Pers Soc Psychol 1996;70:767-79.

72. Chiarotto A, Deyo RA, Terwee CB, et al. Core outcome domains for clinical trials in non-specific low back pain. Eur J Spine 2015;24:1127-42.

73. Deyo RA, Dworkin SF, Amtmann D, et al. Report of the NIH Task Force on research standards for chronic low back pain. Pain Med 2014:15:1249-67.

74. Dworkin RH, Turk DC, Farrar JT, et al. Core outcome measures for chronic pain clinical trials: IMMPACT recommendations. Pain 2005;113:9-19.

75. HIQA. Guidelines for the economic evaluation of health technologies in Ireland. 2014. http://www.hiqa.ie/publication/ guidelines-economic-evaluation-healthtechnologiesireland (accessed 27 Nov 2015)

76. Browne $\mathrm{RH}$. On the use of a pilot sample for sample-size determination. Stat Med 1995;14:1933-40.

77. Lancaster GA, Dodd S, Williamson PR. Design and analysis of pilot studies: recommendations for good practice. J Eval Clin Pract 2004;10:307-12.

78. Shih WJ, Ohman-Strickland PA, Lin Y. Analysis of pilot and early phase studies with small sample sizes. Stat Med 2004;23:1827-42.

79. Lonsdale C, Hall AM, Williams GC, et al. Communication style and exercise compliance in physiotherapy (CONNECT). A cluster randomized controlled trial to test a theory-based intervention to increase chronic low back pain patients' adherence to physiotherapists' recommendations: study rationale, design, and methods. BMC Musculoskelet Disord 2012;13:104.

80. Kerry SM, Bland JM. Statistics notes: The intracluster correlation coefficient in cluster randomisation. BMJ 1998;316:1455-5.

81. Braun V, Clarke V. Using thematic analysis in psychology. Qual Res Psychol 2006;3:77-101.

82. Guerin S, Hennessy E. Pupils' definitions of bullying. Eur J Psychol Educ 2002;17:249-61.

83. Baron RM, Kenny DA. The moderator mediator variable distinction in social psychological-research-conceptual, strategic, and statistical considerations. J Pers Soc Psychol 1986;51: 1173-82.

84. Sobel M. Asymptotic confidence intervals for indirect effects in structural equations models. Sociol Methodol 1982;13: 290-312.

85. Ramsey SD, Willke RJ, Glick $\mathrm{H}$, et al. Cost-effectiveness analysis alongside clinical trials II-an ISPOR good research practices task force report. Value Health 2015;18:161-72. 